\title{
SUPPORTING INFROMATION
}

Hair and Nails as Noninvasive Biomarkers of Human Exposure to Brominated and Organophosphate Flame Retardants

\author{
Liang-Ying Liu, ${ }^{1} \mathrm{Ka} \mathrm{He},{ }^{2}$ Ronald A. Hites,${ }^{1}$ Amina Salamova ${ }^{1, *}$ \\ ${ }^{1}$ School of Public and Environmental Affairs \\ Indiana University \\ Bloomington, Indiana 47405 \\ ${ }^{2}$ School of Public Health \\ Indiana University \\ Bloomington, Indiana 47405
}

Number of pages: 7

Number of Tables: 5 
Table S1. Brominated and organophosphate flame retardants and their abbreviations.

\begin{tabular}{|c|c|}
\hline 2,4-Dibromodiphenyl ether & BDE-7 \\
\hline 2,6-Dibromodiphenyl ether & BDE-10 \\
\hline 4,4'-Dibromodiphenyl ether & BDE-15 \\
\hline 2,2',4-Tribromodiphenyl ether & BDE-17 \\
\hline 2,4,4'-Tribromodiphenyl ether & BDE-28 \\
\hline 2,4,6-Tribromodiphenyl ether & BDE-30 \\
\hline 2,2',4,5'-Tetrabromodiphenyl ether & BDE-49 \\
\hline 2,2',4,4'-Tetrabromodiphenyl ether & BDE-47 \\
\hline 2,3',4,4'-Tetrabromodiphenyl ether & BDE-66 \\
\hline 2,3',4',6-Tetrabromodiphenyl ether & BDE-71 \\
\hline $2,2^{\prime}, 4,4^{\prime}, 5$-Pentabromodiphenyl ether & BDE-99 \\
\hline $2,2^{\prime}, 3,4,4^{\prime}$-Pentabromodiphenyl ether & BDE-85 \\
\hline 2,2',4,4',6-Pentabromodiphenyl ether & BDE-100 \\
\hline 2,3',4,4',6-Pentabromodiphenyl ether & BDE-119 \\
\hline $3,3^{\prime}, 4,4$ ',5-Pentabromodiphenyl ether & BDE-126 \\
\hline 2,2',3,4,4',5'-Hexabromodiphenyl ether & BDE-138 \\
\hline 2,2',3,4,4',6-Hexabromodiphenyl ether & BDE-139 \\
\hline $2,2^{\prime}, 3,4,4^{\prime}, 6^{\prime}-$ Hexabromodiphenyl ether & BDE-140 \\
\hline 2,2',4,4',5,5'-Hexabromodiphenyl ether & BDE-153 \\
\hline 2,2',4,4',5,6'-Hexabromodiphenyl ether + 2,2',4,4',5,5'-hexabromobiphenyl & BDE-154 \\
\hline $2,3,3^{\prime}, 4,4^{\prime}, 5$-Hexabromodiphenyl ether & BDE-156 \\
\hline 3,3',4,4',5,5'-Hexabromodiphenyl ether & BDE-169 \\
\hline 2,2',3,4,4',5,5'-Heptabromodiphenyl ether & BDE-180 \\
\hline $2,2^{\prime}, 3,4,4^{\prime}, 5^{\prime}, 6-$ Heptabromodiphenyl ether & BDE-183 \\
\hline $2,2^{\prime}, 3,4,4$ ',6,6'-Heptabromodiphenyl ether & BDE-184 \\
\hline 2,3,3',4,4',5',6-Heptabromodiphenyl ether & BDE-191 \\
\hline 2,2',3,3',4,4',5,6'-Octabromodiphenyl ether & BDE-196 \\
\hline 2,2',3,3',4,4',6,6'-Octabromodiphenyl ether & BDE-197 \\
\hline $2,2^{\prime}, 3,3^{\prime}, 4,5^{\prime}, 6,6^{\prime}$-Octabromodiphenyl ether & BDE-201 \\
\hline $2,2^{\prime}, 3,4,4$ ',5,5',6-Octabromodiphenyl ether & BDE-203 \\
\hline $2,2^{\prime}, 3,4,4^{\prime}, 5,6,6^{\prime}$-Octabromodiphenyl ether & BDE-204 \\
\hline $2,3,3^{\prime}, 4,4^{\prime}, 5,5^{\prime}, 6-$-Octabromodiphenyl ether & BDE-205 \\
\hline $2,2^{\prime}, 3,3^{\prime}, 4,4^{\prime}, 5,5^{\prime}, 6$-Nonabromodiphenyl ether & BDE-206 \\
\hline $2,2^{\prime}, 3,3^{\prime}, 4,4^{\prime}, 5,6,6^{\prime}-$ Nonabromodiphenyl ether & BDE-207 \\
\hline $2,2^{\prime}, 3,3^{\prime}, 4,5,5^{\prime}, 6,6^{\prime}$-Nonabromodiphenyl ether & BDE-208 \\
\hline Decabromodiphenyl ether & BDE-209 \\
\hline Tetrabromo-p-xylene & pTBX \\
\hline Pentabromobenzene & PBBZ \\
\hline Pentabromoethyl benzene & PBEB \\
\hline Hexabromobenzene & HBB \\
\hline 2-ethylhexyl-2,3,4,5-tetrabromobenzoate & TBB (or EH-TBB) \\
\hline 1,2-Bis(2,4,6-Tribromophenoxy)ethane & TBE (or BTBPE) \\
\hline bis(2-ethylhexyl)-tetrabromophthalate & TBPH (or BEHTBP) \\
\hline syn-Dechlorane Plus & syn-DP \\
\hline anti-Dechlorane Plus & anti-DP \\
\hline tri-n-butylphosphate & TnBP \\
\hline tris(2-chloroethyl)phosphate & TCEP \\
\hline tris(1-chloro-2-propyl)phosphate & TCIPP \\
\hline
\end{tabular}


tris(1,3-dichloro-2-propyl)phosphate

triphenyl phosphate

2-ethylhexyl-diphenyl-phosphate

tris(2-ethylhexyl)phosphate

tri-o-tolyl-phosphate

tri-p-tolyl-phosphate

tris(2-isopropylphenyl) phosphate

tris(3,5-dimethylphenyl)phosphate

tris(4-tert-butylphenyl) phosphate
TDCIPP

TPHP

EHDP

TEHP

TOTP

TPTP

TIPPP

TDMPP

TBPP 
Table S2. Spearman correlation coefficients $(r)$ and probabilities of the significance $(P)$ of the correlation for the levels of major PBDEs, AFRs, and OPEs in hair. Probabilities of significance less than 0.05 are highlighted.

\begin{tabular}{|l|l|l|l|r|r|r|r|r|r|}
\hline & & TDCIPP & TPHP & TBB & TBPH & BDE47 & BDE99 & BDE100 & BDE209 \\
\hline TDCIPP & $r$ & & 0.281 & 0.533 & 0.416 & 0.298 & 0.248 & 0.261 & 0.0638 \\
\hline & $P$ & & 0.0618 & 0.0002 & 0.0058 & 0.0466 & 0.0997 & 0.0826 & 0.6760 \\
\hline TPHP & $r$ & & & 0.376 & 0.499 & 0.289 & 0.316 & 0.313 & 0.205 \\
\hline & $P$ & & & 0.0080 & 0.0004 & 0.0440 & 0.0272 & 0.0285 & 0.1570 \\
\hline TBB & $r$ & & & & 0.529 & 0.427 & 0.347 & 0.346 & -0.0207 \\
\hline & $P$ & & & & 0.0002 & 0.0023 & 0.0148 & 0.0151 & 0.8870 \\
\hline TBPH & $r$ & & & & & 0.393 & 0.365 & 0.349 & 0.0198 \\
\hline & $P$ & & & & & 0.0065 & 0.0118 & 0.0164 & 0.8940 \\
\hline BDE47 & $r$ & & & & & & 0.952 & 0.968 & 0.275 \\
\hline & $P$ & & & & & & 0.0000 & 0.0000 & 0.0531 \\
\hline BDE99 & $r$ & & & & & & & 0.995 & 0.341 \\
\hline & $P$ & & & & & & & 0.0000 & 0.0155 \\
\hline BDE100 & $r$ & & & & & & & & 0.339 \\
\hline & $P$ & & & & & & & & 0.0164 \\
\hline BDE209 & $r$ & & & & & & & & \\
\hline & $P$ & & & & & & & & \\
\hline
\end{tabular}


Table S3. Spearman correlation coefficients $(r)$ and probabilities of the significance $(P)$ of the correlation for the levels of major PBDEs, AFRs, and OPEs in fingernails. Probabilities of significance less than 0.05 are highlighted.

\begin{tabular}{|l|l|l|l|r|r|r|r|r|r|}
\hline & & TDCIPP & TPHP & TBB & TBPH & BDE47 & BDE99 & BDE100 & BDE209 \\
\hline TDCIPP & $r$ & & 0.0956 & 0.0748 & 0.0995 & 0.189 & 0.0949 & 0.129 & 0.374 \\
\hline & $P$ & & 0.6190 & 0.6820 & 0.6040 & 0.2900 & 0.5970 & 0.4700 & 0.0320 \\
\hline TPHP & $r$ & & & 0.0993 & 0.235 & 0.135 & 0.104 & 0.118 & -0.162 \\
\hline & $P$ & & & 0.5560 & 0.1790 & 0.4220 & 0.5390 & 0.4850 & 0.3350 \\
\hline TBB & $r$ & & & & 0.49 & 0.226 & 0.189 & 0.225 & -0.171 \\
\hline & $P$ & & & & 0.0009 & 0.1220 & 0.1970 & 0.1280 & 0.2430 \\
\hline TBPH & $r$ & & & & & 0.133 & 0.0944 & 0.115 & -0.182 \\
\hline & $P$ & & & & & 0.3950 & 0.5450 & 0.4660 & 0.2410 \\
\hline BDE47 & $r$ & & & & & & 0.873 & 0.946 & 0.104 \\
\hline & $P$ & & & & & & 0.0000 & 0.0000 & 0.4720 \\
\hline BDE99 & $r$ & & & & & & & 0.961 & 0.12 \\
\hline & $P$ & & & & & & & 0.0000 & 0.4030 \\
\hline BDE100 & $r$ & & & & & & & & 0.19 \\
\hline & $P$ & & & & & & & & 0.1890 \\
\hline BDE209 & $r$ & & & & & & & & \\
\hline & $P$ & & & & & & & & \\
\hline
\end{tabular}


Table S4. Spearman correlation coefficients $(r)$ and probabilities of the significance $(P)$ of the correlation for the levels of major PBDEs, AFRs, and OPEs in toenails. Probabilities of significance less than 0.05 are highlighted.

\begin{tabular}{|l|l|l|l|r|r|r|r|r|r|}
\hline & & TDCIPP & TPHP & TBB & TBPH & BDE47 & BDE99 & BDE100 & BDE209 \\
\hline TDCIPP & $r$ & & 0.163 & 0.289 & 0.123 & 0.282 & 0.115 & 0.156 & 0.0954 \\
\hline & $P$ & & 0.4630 & 0.1590 & 0.5990 & 0.1690 & 0.5810 & 0.4520 & 0.6460 \\
\hline TPHP & $r$ & & & 0.00283 & 0.0629 & -0.0266 & -0.0434 & -0.0405 & -0.196 \\
\hline & $P$ & & & 0.9860 & 0.7340 & 0.8750 & 0.7970 & 0.8100 & 0.2440 \\
\hline TBB & $r$ & & & & 0.659 & 0.581 & 0.457 & 0.473 & 0.00116 \\
\hline & $P$ & & & & 0.0000 & 0.0000 & 0.0013 & 0.0009 & 0.9940 \\
\hline TBPH & $r$ & & & & & 0.379 & 0.363 & 0.345 & 0.0433 \\
\hline & $P$ & & & & & 0.0161 & 0.0217 & 0.0295 & 0.7890 \\
\hline BDE47 & $r$ & & & & & & 0.946 & 0.959 & 0.229 \\
\hline & $P$ & & & & & & 0.0000 & 0.0000 & 0.1100 \\
\hline BDE99 & $r$ & & & & & & & 0.99 & 0.248 \\
\hline & $P$ & & & & & & & 0.0000 & 0.0822 \\
\hline BDE100 & $r$ & & & & & & & & 0.289 \\
\hline & $P$ & & & & & & & & 0.0440 \\
\hline BDE209 & $r$ & & & & & & & & \\
\hline & $P$ & & & & & & & & \\
\hline
\end{tabular}


Table S5. Spearman correlation coefficients $(r)$ and probabilities of the significance $(P)$ of the correlation for the levels of major PBDEs, AFRs, and OPEs among hair, fingernails, and toenails. Probabilities of significance less than 0.05 are highlighted. The lack of significant correlation for TDCIPP levels between fingernails and toenails is most probably due to the small size of data points (17 pairs).

\begin{tabular}{|l|l|r|r|r|r|r|r|r|r|}
\hline & & \multicolumn{1}{|c|}{ TDCIPP } & \multicolumn{1}{l|}{ TPHP } & \multicolumn{1}{l|}{ TBB } & \multicolumn{1}{l|}{ TBPH } & \multicolumn{1}{l|}{ BDE47 } & \multicolumn{1}{c|}{ BDE99 } & BDE100 & BDE209 \\
\hline Hair-fingernail & $r$ & 0.3760 & 0.3980 & 0.4170 & 0.5360 & 0.5470 & 0.5770 & 0.5750 & 0.6560 \\
\hline & $P$ & 0.0338 & 0.0150 & 0.0034 & 0.0003 & 0.0000 & 0.0000 & 0.0000 & 0.0000 \\
\hline Hair-toenail & $r$ & 0.5310 & 0.3330 & 0.3920 & 0.4390 & 0.5490 & 0.5450 & 0.5290 & 0.4860 \\
\hline & $P$ & 0.0094 & 0.0443 & 0.0066 & 0.0048 & 0.0000 & 0.0000 & 0.0001 & 0.0004 \\
\hline Fingernail-toenail & $r$ & 0.2770 & 0.6330 & 0.6410 & 0.4380 & 0.5440 & 0.6770 & 0.6150 & 0.6090 \\
\hline & $P$ & 0.2750 & 0.0002 & 0.0000 & 0.0069 & 0.0001 & 0.0000 & 0.0000 & 0.0000 \\
\hline
\end{tabular}

